\title{
Negative Poisson's ratios in tendons: An unexpected mechanical response
}

\section{Ruben Gatt ${ }^{a, \Uparrow}$, Michelle Vella Wood ${ }^{a}$, Alfred Gatt b,g, Francis Zarb c, Cynthia Formosa b,g,}

Keith M. Azzopardi ${ }^{a}$, Aaron Casha ${ }^{d}$, Tonio P. Agius ${ }^{e}$, Pierre Schembri-Wismayer ${ }^{d}$, Lucienne Attard ${ }^{f}$, Nachiappan Chockalingam ${ }^{\mathrm{g}, \mathrm{b}}$, Joseph N. Grima ${ }^{\mathrm{a}, \mathrm{h}}$

a Metamaterials Unit, Faculty of Science, University of Malta, Msida MSD 2080, Malta

${ }^{\mathrm{b}}$ Department of Podiatry, Faculty of Health Sciences, University of Malta, Msida MSD 2080, Malta

'Department of Radiography, Faculty of Health Sciences, University of Malta, Msida MSD 2080, Malta

${ }^{d}$ Department of Anatomy, Faculty of Medicine \& Surgery, University of Malta, Msida MSD 2080, Malta

Department of Physiotherapy, Faculty of Health Sciences, University of Malta, Msida MSD 2080, Malta

Department of Orthopaedics, MaterDei Hospital, Msida MSD 2090, Malta

${ }^{\mathrm{g}}$ Faculty of Health Sciences, Staffordshire University, Science Centre, Leek Road ST4 2DF, UK

${ }^{\mathrm{h}}$ Department of Chemistry, Faculty of Science, University of Malta, Msida MSD 2080, Malta 


\section{Abstract}

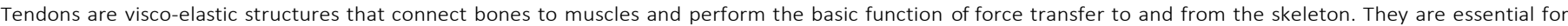

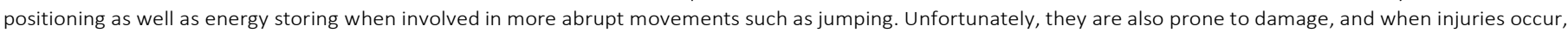

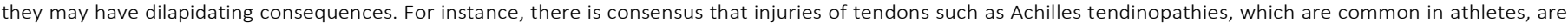

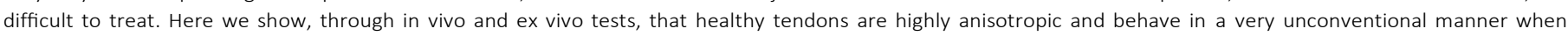

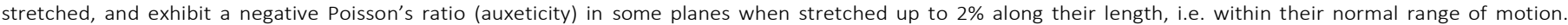

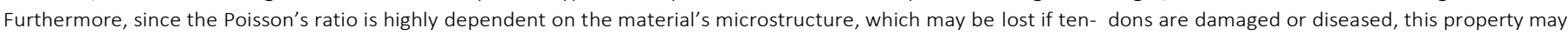
provide a suitable diagnostic tool to assess tendon health.

\section{Statement of significance}

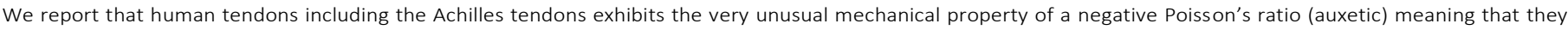

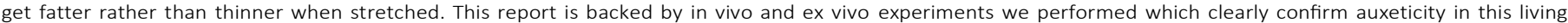

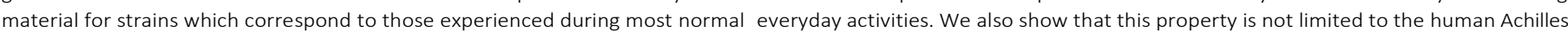

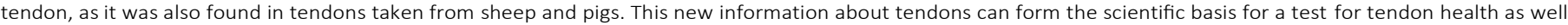
as enable the design of better tendon prosthesis which could replace damaged tendons.

\section{Introduction}

In view of their biological importance, the biomechanical properties of tendons have been the subject of intensive research in recent years, particularly following the early work by Rigby et al.[1] in the mid-twentieth century. Early research described animal models such as rat and horse tendons, with later studies also considering a number of human tendons, particularly those susceptible to injury, such as energy-storing tendons [2]. Most of these studies have focused on their stiffness, where it was reported that tendons appear to adapt their properties in response to the mechanical demands placed on them. Over a period of time, they become stronger and stiffer when subjected to increased stress 
(by repeated strenuous activities), and weaker and less stiff when the stress is reduced [3]. In addition, other factors such as ageing, pregnancy, mobilisation and immobilisation, comorbidities (exam- ples of which can be diabetes mellitus, connective tissue disorders, renal disease), and pharmacologic agents (steroids, non-steroidal anti-inflammatory drugs) are known to affect the biomechanical properties, in particular the stiffness, of tendons [4]. For instance, Stenroth and co-workers [5] reported that a relationship between muscle thickness and cross-sectional area exists, with the older population showing increased tendon thickness and smaller mus- cle size. This hints at altered tendon performance as compared to different muscle size, and suggests tendon compensation for opti- mised locomotion in daily activities.

From a structural perspective, tendons are hierarchical struc- tures: triple helices of tropo-collagen form fibres, which in turn form fibrils, fascicles and eventually tendons. They display a wave-form or crimped structure when relaxed [1], with most recent studies describing it as helical $[6,7]$. Stretching tendons at low strains results in the disappearance of this crimping [8]. This initial deformation corresponds to the 'toeregion' of the stress-strain curve and is followed by the 'elastic region', a zone in which, due to further stretching, the fibres and fascicles slide against each other, eventually returning to their original shape when the load is released. Stretching beyond this range results in permanent deformation [9]. In vivo, tendons usually deform within the toe-region $[10,11]$, with the exception of the energy-storing tendons, which are known to undergo higher strains [12]. This is one of the reasons for the low incidence of injuries to positional tendons when com- pared to energy storing tendons [13].

A few studies have also investigated the Poisson's ratio of ten- dons, both experimentally [2,14-19] and numerically [20,21]. The Poisson's ratio [22] is a fundamental material property in its own accord and describes the change in size of a system in a direc- tion perpendicular to an applied stress. Mathematically, this is defined as the negative of the ratio of the transverse strain to axial strain. Since most materials get thinner (negative strain) when uni- axially stretched (positive strain), one could wrongly assume that the Poisson's ratio is always positive. Nevertheless, it is well known that a negative Poisson's ratio, i.e. the property of getting wider rather than thinner when stretched (auxetic behaviour), is permit- ted by the classical theory of elasticity, with the range of permissi- ble Poisson's ratio for isotropic materials (i.e. having the same properties in all directions) being $16 \mathrm{~m} 60.5$ [23]. This range is even wider for non-isotropic materials. Alegative Poisson's ratio has in fact been found in a wide variety of materials including gra- phene [24], metals [25], foams [26], zeolites [27], silicates [28] and even biological materials such as arteries [29] and skin [30]. Auxeticity in such materials can result in several beneficial fea- tures, ranging from enhanced resistance to indentation to the nat- ural ability to form dome shaped surfaces [31]. Here it must be noted that unlike stiffness, the Poisson's ratio is a twodimensional property and in anisotropic materials, its sign and magnitude may depend not only on the direction of stretching, but also on the orthogonal direction being measured. Unfortunately, in view of the complexity associated with studying the Poisson's ratios, studies reporting this property in tendons have been limited in number [2,14-19], and normally make various assumptions, which may have resulted in incomplete reporting of the Poisson's ratio. For example, in most ex vivo studies on the Poisson's ratios of tendons it had been assumed that the tendon exhibits transverse isotropy, with reported values typically ranging between 0.4 and $4.3[2,14-17]$ when the Poisson's ratio is mea- sured in the elastic region.

In this paper we show, through both ex vivo and in vivo studies, that healthy tendons of both human and animal origin have a neg- ative Poisson's ratio when stretched along their length, in the plane

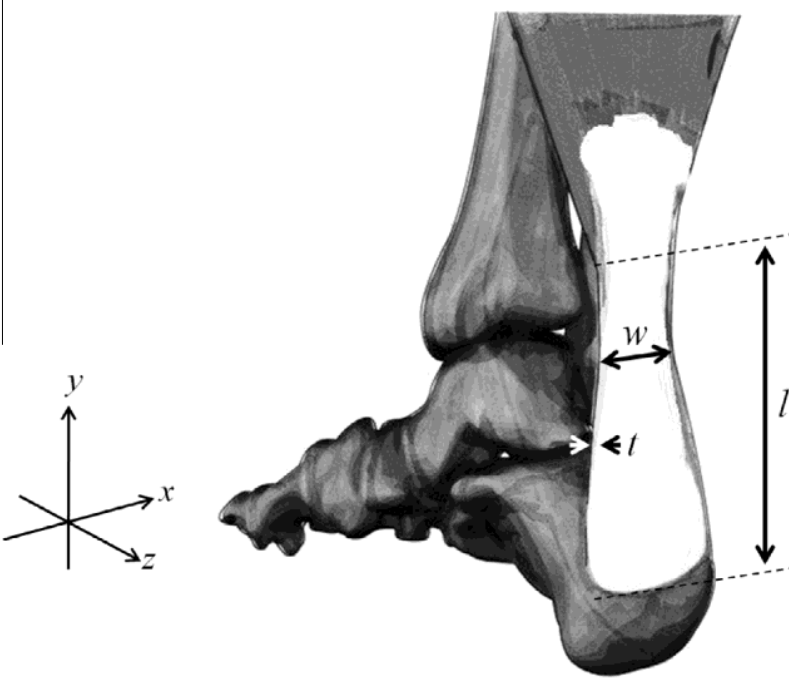

Fig. 1. Figure showing the different parameters used to define the various tendon dimensions in this study. Note that the $x y$ plane is equivalent to the coronal plane (this being the plane where auxeticity was measured), the $y z$ plane is equivalent to the sagittal plane (this being the plane where conventional Poisson's ratio was measured), and the $x z$ plane is equivalent to the axial plane.

of the width of the tendon, i.e. for example the coronal plane in the case of the Achilles tendon (see Fig. 1).

\section{Materials and methods}

\subsection{Ex vivo experiments}

Ex vivo experiments were carried out on a number of tendons: human, pig and sheep in origin. The human tendons were obtained from cadavers donated to the Anatomy Department of the University of Malta, whilst sheep and pig tendons were obtained from a local abattoir. More specifically, in the case of the human samples, the mechanical properties at room temperature of the Achilles and Peroneus brevis tendons, obtained from human fresh frozen cadaveric tissue, were measured in this study. Two samples were tested, due to the difficulty of acquiring such tissues. In the case of the animal samples, the mechanical properties of the deep flexor tendon were measured on five samples from each species. These samples were obtained and tested within $24 \mathrm{~h}$ of the animal death, and were kept in a refrigerator at $5{ }^{\circ} \mathrm{C}$ until the tests were carried out.

The tendons were first dissected to remove connective tissue and surrounding sheaths. The ends of the tendons were wrapped with nylon cord prior to clamping, with the aim of preventing the tendon from expanding laterally within the clamp on tighten- ing. Additionally, the size of the clamp itself was just large enough to fit the tendon and nylon wrapping with no space for further expansion. The clamps used were as those used by Fessel et al. [32]. The Poisson's ratios of the tendons were tested using a tensile loading machine (Testometric, UK) having a $100 \mathrm{~kg} \mathrm{~F}$ load cell (S/N 31,931), equipped with a duly calibrated camera videoextensometer (Messphysik, Germany). Before the actual test phase, a preconditioning step was applied. This involved stretching the tendon until a force of $1 \mathrm{~N}$ was reached, holding this force for $30 \mathrm{~s}$ and then returning to $0 \mathrm{~N}$. The cycle was repeated for a total of 10 times. Tests were carried out using strain rate control at a rate of $5 \mathrm{~mm} / \mathrm{min}$. Measurements, through video extensometry, were taken for the length (I) and width $(t)$ of the tendons, which were appropriately marked for the Messphysik pattern recognition software, as shown in Fig. 2. The pattern recognition protocol 


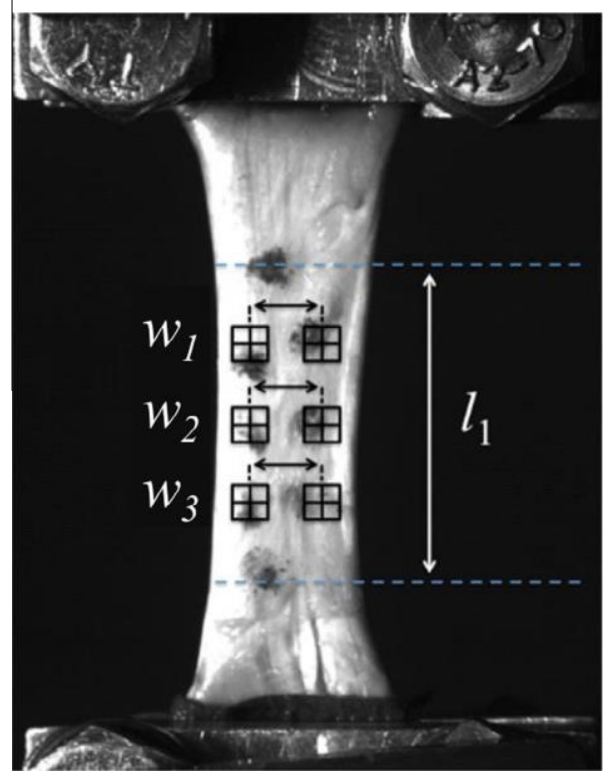

Fig. 2. The placement of the tendons in the tensile loading machine, together with the markings used to measure the axial and longitudinal strains. Note that the dark cloudy spots are the markings used. The squares indicate the region where the software is tracking the pattern whilst the lines indicate the distance between the markings.

operates by establishing memory zones around each target where the grey scale level are recorded. These memory zones are dynam- ically adapted throughout the testing not to lose the target if there are changes in the markings themselves.

In the main set of measurements, aimed to measure the Poisson's ratio in the plane which is parallel to the surface of the bone next it, three transverse widths $\left(w_{1}-w_{3}\right)$ and one axial length $\left(I_{1}\right)$ were recorded for each tendon. As much as possible, the trans- verse width measurements were taken from the centre of the spec- imen in order to reduce any edge effects present. The axial length $\left(/_{1}\right)$ was measured from the tendon itself (see Fig. 2) in order to avoid erroneous reading due to slippage and damage at the ten- don-clamp interface [33-36]. Experiments were run up to $2 \%$ axial strain. The samples were wetted with saline water at regular inter-vals throughout the tests to keep them moist.

Furthermore, from an additional five samples of pig deep flexor tendons, the Poisson's ratio in the plane which is orthogonal to the one above, i.e. the plane which is orthogonal to the surface of the bone next it, three transverse thicknesses $\left(t_{1}-t_{3}\right)$ and one axial length $\left(I_{1}\right)$ were recorded for each tendon.

For each sample tested, all three readings of the transverse dimensions vs axial dimensions were used in the data analysis and averaged as follows: for each of the three sets of 'transverse dimensions vs axial dimensions' measured, a sixth order polyno- mial was first fitted and used to smoothen the data, and hence obtain three sets of smoothened 'transverse dimensions vs axial dimensions'. The engineering transverse strains were then calcu- lated for each of the three sets of smoothened data, averaged, and plotted against the axial strain. This results in a single engi- neering transverse strain vs axial strain (plotted in Fig. 4), from which the engineering Poisson's ratio up to $0.3 \%$ axial strain was calculated as the negative of the slope of the graph, assuming lin- earity in the initial $0.3 \%$ axial region. For the initial Poisson's ratios, the square of the Pearson product moment correlation coefficient $\left(R^{2}\right)$ was also calculated.

For all of the samples tested, the incremental Poisson's ratio [37], also known as the Poisson's function [38] was also calculated. This form of the Poisson's ratio gives a much better indication of changes in the lateral dimension of the sample as a measure of applied strain, since it takes into consideration the fact that upon loading, the shape of the material under investigation (the tendon in this case) may change dynamically in a non-linear manner. This incremental Poisson's ratio in the $x y$-plane for loading in the $y$ direction may be defined as:

$\mathrm{m}_{y x}^{\text {inc }}{ }^{1 / 4-} \mathrm{de}^{\frac{\mathrm{de}_{x}^{\mathrm{inc}} y}{\mathrm{inc}}}$

where, referring to Fig. 1 , de $\mathrm{ein}^{\mathrm{inc}}$ is the incremental strain in the $x$-direction (width of the tendon), and de $\mathrm{e}^{\mathrm{inc}}$ is the incremental strain in the $y$-direction (length of the tendon) which strains may be obtained as below:

$\mathrm{de}_{x} 1 / 4 \frac{\left(n \beta Y^{\text {avg }}-W^{\text {avg }}\right.}{w_{n}^{\text {avg }}} ; \mathrm{de}_{y} 1 / 4 \quad \frac{(-1}{{ }_{n \mathrm{p} 1}{ }_{n}}$

where $I_{n}, I_{n+1}$ are sequential readings of the axial dimension, and $W_{n}, W_{n+1}$ are the corresponding sequential width readings in the trans- verse dimension (averaged over the three measurement lines). Note that in this case, the data was read from the smoothened measure- ments at intervals, which correspond to $\mathrm{de}_{y} \frac{1}{4}$ 0:062\%. Similar argu- ments can be made for $\mathrm{m}^{\text {inc }}$, the incrementstal Poisson's ratio in the $y z$-plane for loading in the $y$ direction.

\subsection{In vivo experiments}

In vivo experiments were carried out on the left leg Achilles ten- don of two individuals, using Magnetic Resonance Imaging (MRI) (Gyroscan, Philips, (1.5 T), Netherlands) in conjunction with a ded-icated ankle/foot quadrature coil. Subject M1 was a male aged 25 whilst subject M2 was a female aged 33. Both subjects are physi- cally active. The Research Ethics committee of the University of Malta approved the study on the 20th October 2014 and the sub- jects provided informed consent.

Proton density weighted turbo spin echo (PDW TSE) axial scans with 2 $\mathrm{mm}$ slice thickness, the limit of the machine, were used in both cases with no gaps between the slices. Two foot positions were tested, the neutral position and the dorsiflexion position (using the maximum amount of tension that the subject could hold). The following scan parameters were selected for the PDw TSE sequences: time to repeat (TR) - $3000 \mathrm{~ms}$; time to echo (TE)

- $30 \mathrm{~ms}$; band width $-184.3 \mathrm{~Hz}$; number of signal averages (NSA) -4 . The field of view (FoV) settings were as follows: right to left (RL) -250 $\mathrm{mm}$; anterior to posterior (AP) $-250 \mathrm{~mm}$ and feet to head (FH) $-120 \mathrm{~mm}$. The MRAcquisition Frequency Encoding Step was 532 whilst the MRAcquisition Phase Encoding steps in-plane was 420. This result in voxels having the size of $470 \mathrm{Im}$ by $600 \mathrm{Im}$ by $2000 \mathrm{Im}$. Note that the testing time with these set- tings resulted in a data collection phase of ca. $30 \mathrm{~min}$ for each foot position. In order for the subjects not to move their feet during this time, the position of the foot was secured in the ankle quadrature coil using pieces of foam. Furthermore, sand bags were positioned on the lower portion of the leg to reduce any movement.

Measurements of the length and width of the tendons were performed using the Osirix 32-bit software (Pixmeo SARL, Switzerland) [39]. An estimation of the length of the tendon (both at the neutral position and at the dorsiflexed position) was made by reconstructing sagittal slices of the tendon from the axial data obtained through the scans, using an inbuilt feature of the Osirix software. The starting point of the length measurement was taken at the point of attachment of the Achilles tendon to the Gastrocnemius muscle (tendon muscle junction (TM) in Fig. 3), while the ending point of the measurement was taken at the point of attachment of the Achilles tendon to the calcaneus (tendon bone junction (TB) in Fig. 3). The width of the tendon was taken from the axial dataset, along the length of the Achilles 

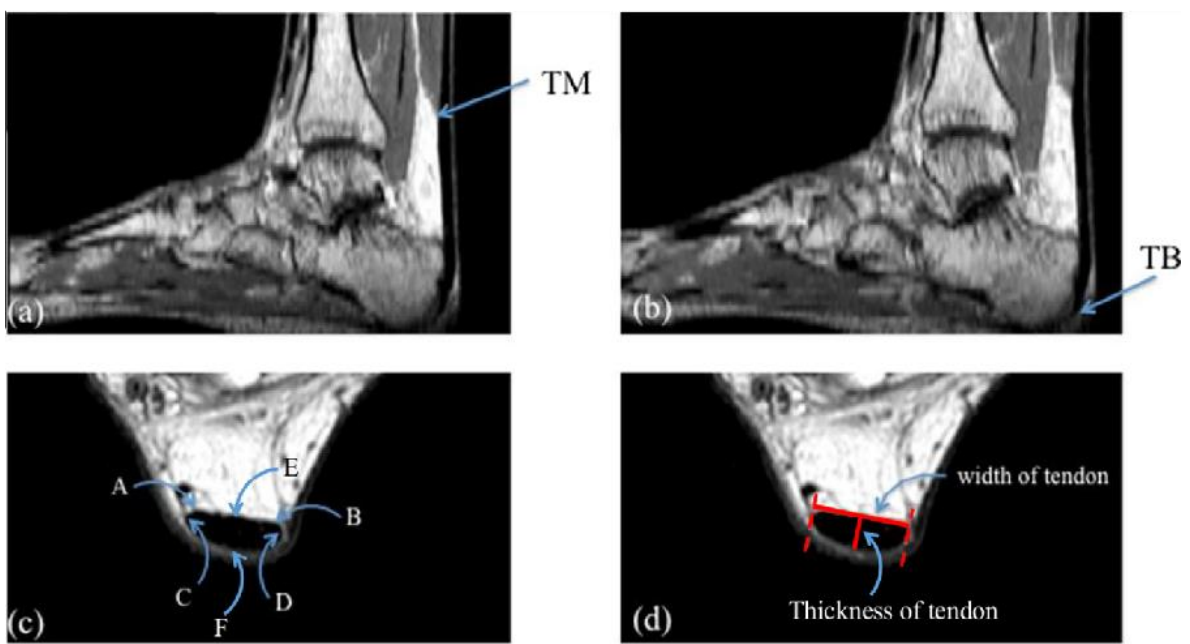

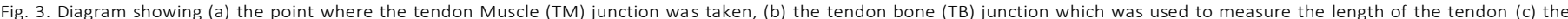

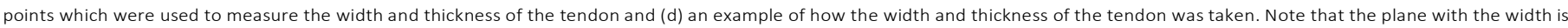
the plane where auxeticity was measured whilst the plane with the thickness is the plane where conventional Poisson's ratio was measured.
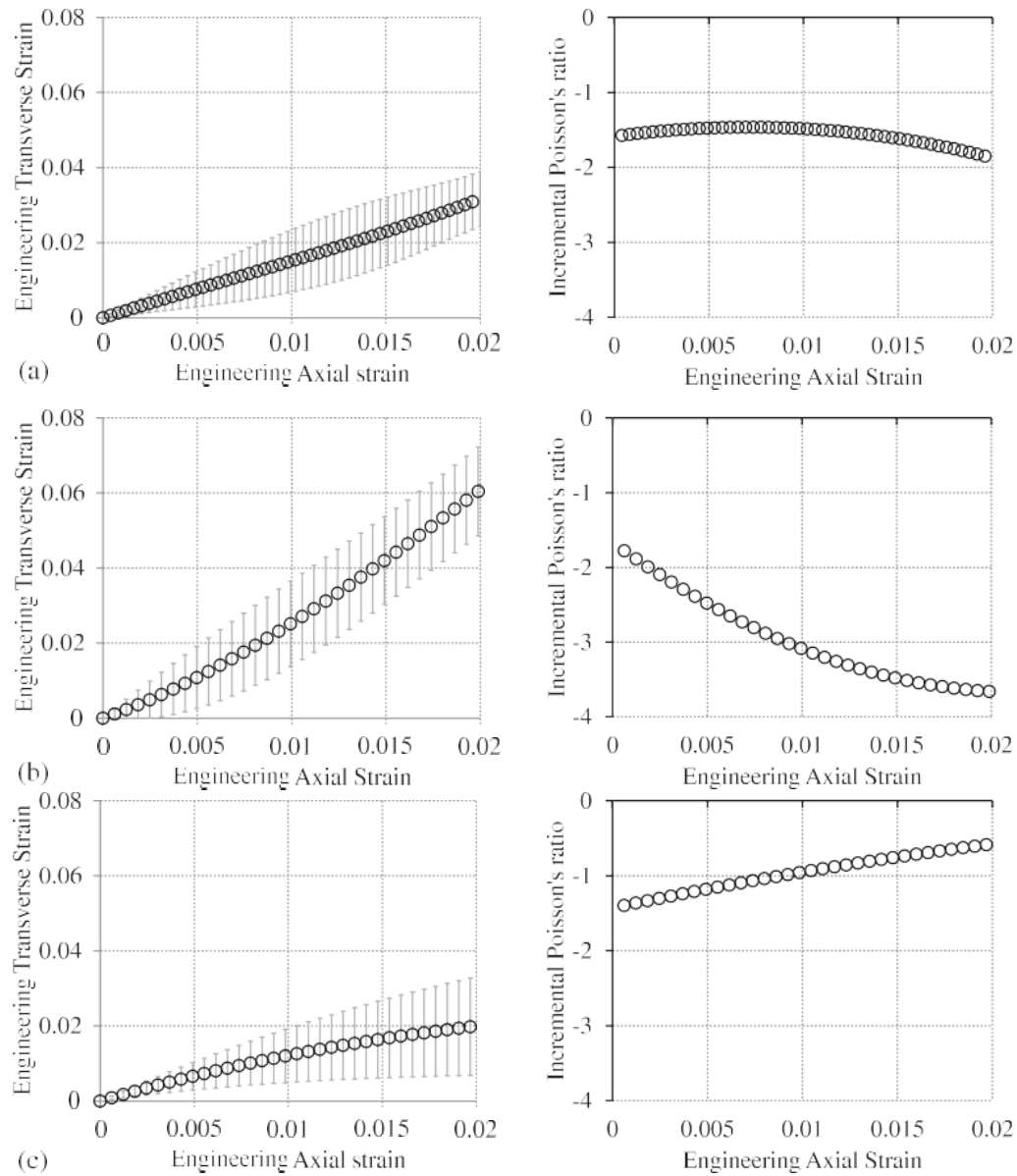

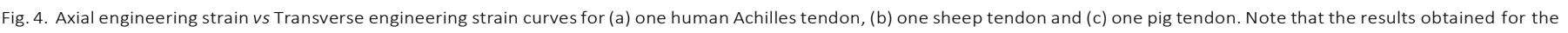
Peroneus brevis and more in-depth results are presented in the Supplementary information.

tendon, starting below the tendon-muscle aponeurosis up to the point where the calcaneus starts touching the tendon. The points closest to the tibia and fibula in the left and right sides of the Achilles tendon (points A and B in Fig. 3) were first identified and the width of the tendon was taken to be the distance between the leftmost part of the tendon, marked as point $C$ in Fig. 3, and the rightmost part of the tendon, marked as point $D$ in the same Figure, when projected onto a line drawn parallel to points A and B.

From the same set of MRIs, a similar set of measurements were also recorded so as to quantify the change in thickness of the 
Table 1

The Negative Poisson's ratio measured in the plane parallel to the surface of the bone for the initial extension ( $0.3 \%$ strain) of the tendons tested.

\begin{tabular}{llll}
\hline Tendon & Sample & Poisson's ratio & $R^{2}$ \\
\hline Achilles & HA1 & -1.44 & 0.99 \\
& HA2 & -0.39 & 0.78 \\
Peroneus brevis & HPB1 & -3.81 & 0.96 \\
& HPB2 & -0.166 & 0.99 \\
Deep flexor (sheep) & SDF1 & -2.23 & 0.99 \\
& SDF2 & -1.14 & 0.99 \\
& SDF3 & -0.37 & 0.93 \\
& SDF4 & -3.56 & 0.99 \\
& SDF5 & -9.86 & 0.99 \\
Deep flexor (pig) & PDF1 & -0.56 & 0.99 \\
& PDF2 & -0.34 & 0.80 \\
& PDF3 & -3.11 & 0.99 \\
& PDF4 & -1.32 & 0.99 \\
& PDF5 & -1.32 & 0.99 \\
\hline
\end{tabular}

tendon for stretching along the length of the tendon. For these measurements, referring to Fig. 3, the thickness of the tendon was measured as the distance $E$ to $F$.

Note that since the position of the tendon changes relative to the tibia and fibula (it is being stretched downwards), it is impor-tant to take the measurements of the width and thickness in such a way that the same part of the tendon is being measured between the neutral position and the tensioned (dorsiflexed) position. This was achieved by starting the measurements exactly below the muscle aponeurosis in each case.

\section{Results}

\subsection{Ex vivo}

Plots showing the axial engineering strain versus the average transverse engineering strain and the incremental Poisson's ratio versus the axial engineering strain for the plane parallel to the sur-face of the bone are shown in Fig. 4 and in the Supplementary information. Also shown in the Supplementary information are plots showing the equivalent data for the plane orthogonal to the surface of the bone as measured in the five pig deep flexor tendons. Furthermore, a table with the initial Poisson's ratios (up to $0.3 \%$ ) are presented for each sample tested (see Table 1 and Table 2). This data suggest that the tendons tested are highly anisotropic at low strains and exhibit some degree of auxetic behaviour in the plane parallel to the surface of the bone within the strain region tested, which corresponds to the strains at normal activity. Unfortunately, to our knowledge, this important finding was never reported even if it may have had been observed by other research-ers in the past. For example, in the literature, it is reported that inappropriate clamping of tendons may give wrong results, whereby the presence of residual stresses may erroneously give a negative measurement of the Poisson's ratio, suggesting that aux- eticity in tendons could have indeed been observed by Shadwick more than two decades ago but was overlooked as an artefact [40]. In this study, prior to clamping, the ends of the tendons were wrapped with nylon cord and the clamp was just large enough to fit the tendon and nylon wrapping with no space for further expan- sion. This clamping set-up should minimise the risk of having com- pressive forces which could otherwise result in unwanted deformations due to clamping.

Another interesting observation is that the actual shape of strainPoisson's ratio curves among Human tendon, sheep and pig in Fig 4 seem to be specie-specific. It is beyond the scope of this work to identify what gives rise to these differences but is more
Table 2

The positive Poisson's ratio measured in the plane orthogonal to the surface of the bone for the initial extension ( $0.3 \%$ strain) of the tendons tested.

\begin{tabular}{llll}
\hline Tendon & Sample & Poisson's ratio & $R^{2}$ \\
\hline Deep flexor (pig) & PDF6 & 3.035 & 1.00 \\
& PDF7 & 1.415 & 1.00 \\
& PDF8 & 1.217 & 1.00 \\
& PDF9 & 1.637 & 1.00 \\
& PDF10 & 1.437 & 1.00 \\
\hline
\end{tabular}

than likely that a multitude of factors, ranging from evolution to the lifestyle could have an effect.

\subsection{In vivo}

In these experiments, two MRI sequences were taken, the first one for the foot in a neutral position and the second one for the foot in a dorsi-flexed position. Upon dorsiflexion, the calcaneus which is the distal attachment of the Achilles' tendon is displaced downwards (distally) as the talus hinges in the ankle joint, which in turn stretches the Achilles tendon-muscle system. From the scans obtained, it was evident that the width of the tendon in the plane parallel to the surface of the bone (in this case the coro- nal plane) increased on stretching due to dorsiflexion, confirming auxetic behaviour (see Fig. 5). Here it must be emphasised that the observation that a negative Poisson's ratio, present in this plane, was identifiable since the in-plane resolution (cross- sectional) was high enough to measure the width and thickness of the tendon, but not easy to quantify, since the extent of stretch-ing of the Achilles tendon (along the length of the tendon) was low and comparable to the slice thickness. The stretching of the tendon was accompanied by a much larger extension of the gastrocnemius muscle which stretched by ca. $6 \mathrm{~mm}$ and $8 \mathrm{~mm}$ respectively. Nevertheless, even if the muscle stretched more than the Achilles tendon, the Achilles tendon was always observed to stretch, thus permitting this in vivo verification that the Poisson's ratios is neg- ative upon stretching. These scans, see data in Supplementary information, also confirmed a positive Poisson's ratio in the orthog- onal plane even at the small strains measured here which confirm results by others who also measure positive Poisson's ratios in such planes. This also highlights the highly anisotropic nature of tendons.

\section{Discussion}

The work being reported here, i.e. that healthy tendons of both animal and human origin exhibit auxetic behaviour suggests that nature has found a manner in which to achieve this highly anoma- lous mechanical response even within living tissues. This in itself is a very important finding from the purely scientific point of view since it is well known that a negative Poisson's ratio is not that commonly encountered in most everyday materials.

Nevertheless, this result should not be considered as a mere sci- entific curiosity since our finding that tendons should have a neg- ative Poisson's ratio has numerous important implications. Knowledge that healthy tendons exhibit a negative Poisson's ratio in particular planes should help in any study looking at developing synthetic allografts to replace injured tendons, since it is accepted that for optimal performance the prosthesis should have similar properties to the tissue it is replacing. A number of studies have also shown that unhealthy tendons tend to lose their structure, mainly their crimping [41], which as discussed below may be an essential feature for auxeticity. In fact, Järvinen et al. noted that unhealthy tendons present a reduced crimp angle, crimp continu- ity and reduced fibre diameter [41]. Since, the Poisson's ratio is 

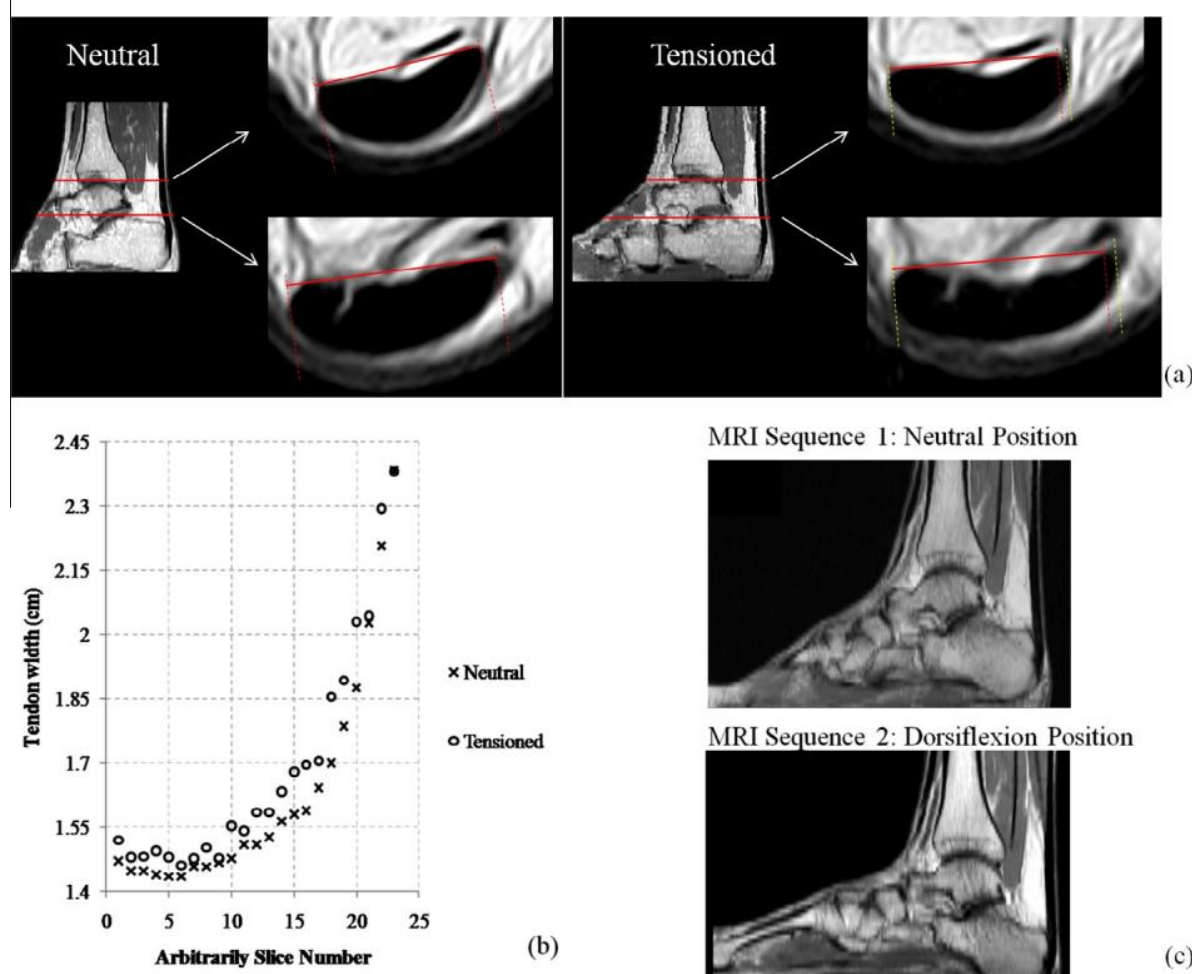

(a)

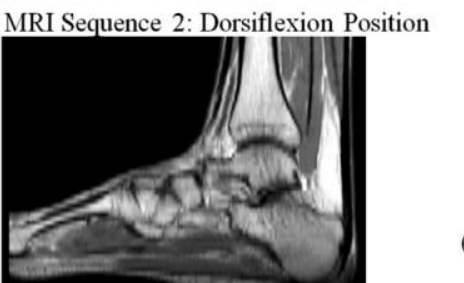

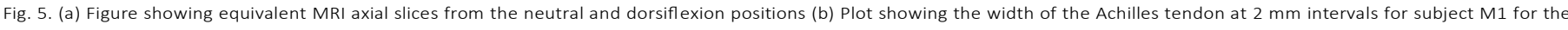
neutral and tensioned positions (c) MRI sagittal slice showing the positions of the foot for the first MRI sequence (neutral position) and the second MRI sequence (dorsiflexion position).

normally associated with a particular geometry and the way this structure deforms, we are proposing that the Poisson's ratio may be used as an indicator of tendon health. This concept may have health and financial implications, since it may present a way to monitor potential tendon injuries and detect early tendon changes (before there is an actual clinical injury). This is especially useful in persons performing highly demanding physical activities, such as top athletes and professional football players, who frequently suf- fer from tendon injuries such as Achilles, patellar, quadriceps and hamstring tendinopathies. It may also prove useful in animals, such as race-horses, which are prone to tendon injuries due to the high demands of racing. Serial MRI studies may be used as new diagnostic testing technique in which tendons with suspected afflictions have their Poisson's characteristics analysed to provide the clinician with new, and perhaps more specific, markers of ten- don health. They can potentially be used to document progress in tendon repair by assessing the return of the negative Poisson's ratio (after confirming that injured tendons do in fact regain a neg- ative Poisson's ratio), since tendons have prolonged recoveries because of their poor blood supply [42]. Such hypotheses may only be confirmed after further in-depth studies utilising both healthy and diseased tendons have been performed.

It is beyond the scope of this work to provide an explanation for the causes of auxeticity, as this requires further in-depth studies. However, in light of the fact that auxeticity was measured in the toe-region of the stress-strain curve, where tendons are reported to have a crimped structure, [43] it may be hypothesised that this feature of tendons is likely to play a role in the generation of this observed anomalous behaviour. This may be further corroborated when taking into consideration the works by Raspanti et al. [44], Hansen et al. [8] and Grima et al. [24] together. Raspanti and co-workers showed that the crimps in tendons do not follow a smooth wave, but the fibrils tend to change direction abruptly resulting in large discontinuities. This crimp structure is similar to the crumpled paper model, which was used by Grima et al. to describe auxetic behaviour in graphene having defects. Further to this, Hansen and co-workers showed that upon elongation, the crimp structure in tendons straightens from the outside to the cen- tre of the fascicle. Taken together, these observations do suggest that upon loading, the crimp structure deforms in a way similar to a crumpled paper resulting in the observed negative Poisson's ratio. Obviously such auxeticity in one of the planes is necessarily accompanied by a positive Poisson's ratio in the orthogo nal plane, a property which is not only characteristic of tendons we tested which were found to be auxetic in the coronal plane and non-auxetic in the orthogonal plane, but also in other systems which exhibit auxeticity as a result of unfolding of wavy structures including graphene and crushed papers [24], an easily visible effect as illustrated in the Supplementary information (ANIM1 showing the in-plane auxetic behaviour and ANIM2 showing the out of plane positive Poisson's ratio). Such anisotropy has also been found in other biological materials such as skin, highlighting the impor- tance of testing the mechanical properties in more than one direc- tion or plane.

Furthermore, auxeticity in these bio-structures is most proba- bly an evolved trait. This is because negative Poisson's ratios may give tendons a number of advantages, which result in better func- tionality. For example, tendons have some viscoelastic character [45], i.e. the presence of auxeticity may also enhance their damp-ing capability by changing stresses and deformations present within the tendon as a function of time [46].

Also, the plane of the tendon which is auxetic faces the surface of the body and is parallel to the surface of the bone. Thus, as ten- dons are stretched, the area in contact with the bone increases sig- nificantly in a much more pronounced manner than if the Poisson's ratio was positive, with the added benefit there is less stress at the 


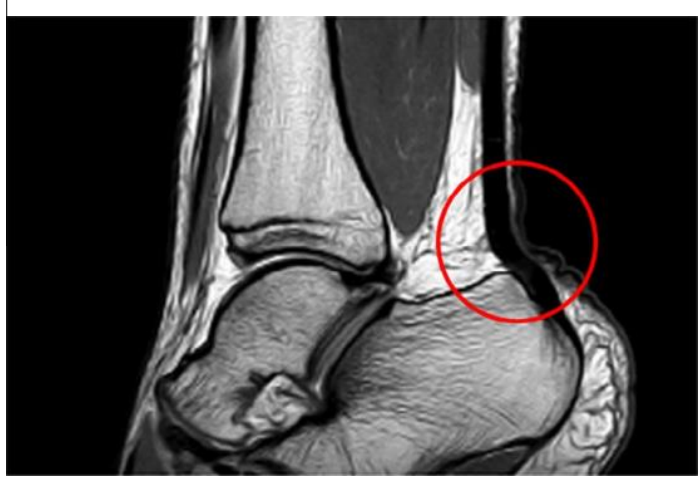

Fig. 6. MRI sagittal slice showing how the tendon 'kinks' when subjected to active plantar flexion.

points of contact between the tendon and the underlying structures.

Furthermore, a negative Poisson's ratio imparts on tendons a natural ability to adopt synclastic curvatures, a highly desirable property since tendons often have to wrap around dome-shaped bones. For example, the Achilles tendon is constrained to adopt a synclastic curvature every time that it is stretched during plantar flexion of the foot where it has to wrap around the dome-shaped calcaneum. Had the Achilles tendon not been auxetic, much higher internal stresses would have been generated in this tendon as it bends around the calcaneum, with the obvious undesirable conse- quences such added internal stresses bring with them.

Before concluding it is important to note some of the limitations of this study. The main one is that the in vivo tests could only be carried out in a qualitative manner and that the tendons tested could only be stretched by a small extent making the lateral exten- sion difficult to quantify, even if this was enough for a qualitative interpretation. It may be argued that if the subjects were subjected to active plantar flexion, the tendon would stretch to a higher degree since the muscle would pull it directly. Although this may be true, at high plantar flexion the rotation of the calcaneus would cause the Achilles tendon to kink as shown in Fig. 6, something which, unless catered for, may result in erroneous results. It is also important to note that in vivo data is much harder to interpret since more than one factor may be at play at any given moment. In fact, upon dorsiflexion (as in the case of this study), the tendon may be subjected to loads from different directions, at least in the aponeurosis, as this part is directly attached to the muscle, which changes shape upon contraction or extension. However, the degree of transverse loading of the aponeurosis is not easy to quantify since it depends on its stiffness relative to that of the muscle. In the case of our study it was noted that the change in width of the tendons tends to increase as one moves away from the aponeu-rosis, where the effect of transverse loading is diminished.

It should also be mentioned that the present work is focusing on small strain deformations of tendons, i.e. ones which are less than $2 \%$ and which correspond to what one normally achieves in normal everyday activities, and in specific planes. Thus one should be care- ful when interpreting the results being reported in this work and comparing them to work of others. For example, the results obtained by Obst et al. [18] and Iwanuma et al. [19] who found that upon maximal voluntary isometric contraction of the plantarflex- ors, the width of the tendon decreases. This finding cannot be com- pared directly to what we are reporting here and may be explained by the fact that at such contraction level, the Achilles tendon, which is an energy storing tendon, would not be in the toe-region, but instead, it would be in the elastic region, where the crimping of the tendon would be exhausted and deformation would occur by the sliding of fibres. All this further suggests that crimping may have an important role in the observed negative Poisson's ratios.

\section{Conclusion}

To conclude, in this work we have reported that animal ten- dons, including human tendons, exhibit a negative Poisson's ratio (auxetic behaviour), a property which imparts several beneficial properties in such a way to optimise their behaviour. This finding was confirmed by ex vivo testing on human Achilles and Peroneus brevis tendons, and the deep flexor tendon from pig and sheep. In vivo studies were also carried out on the Achilles ten- dons of two individuals, where it was found that these tendons show a negative Poisson's ratio for plantar flexion. It is hypothesised that such a property may be lost if the tendons are in some way damaged. This is the first time that these tendons are being characterised in this way. If further investigations are carried out, these findings are likely to be of significance to clinical use as a possible diagnostic measure of the state of health of tendons, as well as to scientists who may be working on development of pros- thetic tendons. We also hope that this paper will stimulate further experimental and modelling work, which would give a clearer pic- ture on how nature achieves the phenomenon of negative Poisson's ratios in human and animal tendons. This may provide a blue-print for the design of new man-made auxetic materials and metamate- rials which mimic the behaviour of these biological systems [47]. 
Competing financial interest statement

The authors have no competing financial interests.

Acknowledgements

Part of this work has been funded by the Malta Council for Science and Technology through the R\&I-2012-061 Project (SMESH). The Authors would also like to acknowledge Kieran Chircop, who performed ultra-sound tests in a preliminary study which led to this paper and St. James Hospital Malta for giving per-mission for the utilisation of their MRI equipment.

Appendix A. Figures with essential colour discrimination

Certain figures in this article, particularly Figs. 2, 3, 5 and 6, are difficult to interpret in black and white. The full colour images can be found in the on-line version, at http://dx.doi.org/10.1016/j.act- bio.2015.06.018.

Appendix B. Supplementary data

Supplementary data associated with this article can be found, in the online version, at http://dx.doi.org/10.1016/j.actbio.2015.06. 018.

References

[1] B.J. Rigby, N. Hirai, J.D. Spikes, H. Eyring, The mechanical properties of rat tail tendon, J. Gen. Physiol. 43 (1959) 265-283.

[2] C.T. Thorpe et al., Helical sub-structures in energy-storing tendons provide a possible mechanism for efficient energy storage and return, Acta Biomater. 9(2013) 7948-7956.

[3] F.R. Noyes, Functional properties of knee ligaments and alterations induced by immobilization: a correlative biomechanical and histological study in primates, Clin. Orthop. Relat. Res. 123 (1977) 210-242.

[4] N. Nordin, V. Frankel, Basic Biomechanics of the Musculoskeletal System, Lippincott Williams and Wilkins, Pennsylvania, 2012.

[5] I. Stenroth, J. Peltonen, N.J. Cronin, S. Sipilä, T. Finni, Age-related differences in Achilles tendon properties and triceps surae muscle architecture in vivo, J. Appl. Physiol. 113 (2012) 1537-1544.

[6] S.P. Reese, S.A. Maas, J.A. Weiss, Micromechanical models of helical superstructures in ligament and tendon fibers predict large Poisson's ratios, J. Biomech. 43 (2010) 1394-1400.

[7] C. Vidal Bde, M.L. Mello, Structural organization of collagen fibers in chordae tendineae as assessed by optical anisotropic properties and Fast Fourier transform, J. Struct. Biol. 167 (2009) 166-175.

[8] K.A. Hansen, J.A. Weiss, J.K. Barton, Recruitment of tendon crimp with applied tensile strain, J. Biomech. Eng. 124 (2002) 72-77.

[9] M. Benjamin, E. Kaizer, S. Milz, Structure-function relationships in tendons: a review, J. Anat. 212 (2008) 211228.

[10] C.N. Maganaris, J.P. Paul, In vivo human tendon mechanical properties, J. Physiol. 521 (1999) 307-313.

[11] C.N. Maganaris, J.P. Paul, Tensile properties of the in vivo human gastrocnemius tendon, J. Biomech. 35 (2002) 1639-1646.

[12] G.A. Lichtwark, A.M. Wilson, In vivo mechanical properties of the human Achilles tendon during one-legged hopping, J. Exp. Biol. 208 (2005) 4715-4725.

[13] H.L. Birch, A.M. Wilson, A.E. Goodship, Physical activity: does long-term, high- intensity exercise in horses result in tendon degeneration?, J Appl. Physiol. 105 (2008) 1927-1933.

[14] H.A. Lynch, W. Johannessen, J.P. Wu, A. Jawa, D.M. Elliott, Effect of fiber orientation and strain rate on the nonlinear uniaxial tensile material properties of tendon, Trans. ASME 125 (2003) 726-731.

[15] C.T. Thorpe, G.P. Riley, H.L. Birch, P.D. Clegg, H.R.C. Screen, Effect of fatigue loading on structure and functional behaviour of fascicles from energy-storing tendons, Acta Biomater. 10 (2014) 3217-3224.

[16] C. Vergari et al., True Stress and Poisson's ratio of tendons during loading, J. Biomech. 44 (2011) 719-724.

[17] L.A. Chernak, D.G. Thelen, Tendon motion and strain patterns evaluated with two-dimensional ultrasound elastography, J. Biomech. 45 (2012) 2618-2623.

[18] S.J. Obst, R. Newsham-West, R.S. Barrett, In vivo measurement of human Achilles tendon morphology using freehand 3-D ultrasound, Ultrasound Med. Biol. 40 (2014) 62-70. 
[19] S. Iwanuma et al., Longitudinal and transverse deformation of human Achilles tendon induced by isometric plantar flexion at different intensities, J. Appl. Physiol. 110 (2011) 1615-1621.

[20] S.P. Reese, J.A. Weiss, Tendon fascicles exhibit a linear correlation between Poisson's ratio and force during uniaxial stress relaxation, J. Biomech. Eng. 135 (2013) 034501.

[21] L. Yin, D.M. Elliott, A biphasic and transversely isotropic mechanical model for tendon: application to mouse tail fascicles in uniaxial tension, J. Biomech. 37 (2004) 907-916.

[22] L.D. Landau, E.M. Lifshitz, A.M. Kosevich, I.P. Pitaevskii, Theory of Elasticity, Pergamon Press, London, 1986.

[23] Y.C. Fung, Foundation of Solid Mechanics, Prentice-Hall, New Jersey, 1968, p. 353.

[24] J.N. Grima et al., Tailoring graphene to achieve negative Poisson's ratio properties, Adv. Mater. (2014), http://dx.doi.org/10.1002/sdma.201404106.

[25] R.H. Baughman, J.M. Shacklette, A.A. Zakhidov, S. Stafstrom, Negative Poisson's ratios as a common feature of cubic metals, Nature 392 (1998) 362-365.

[26] R. Lakes, Foam structures with a negative Poisson's ratio, Science 235 (1987) 1038-1040.

[27] J.N. Grima, R. Jackson, A. Alderson, K.E. Evans, Do zeolites have negative Poisson's ratios?, Adv Mater. 12 (2000) 1912-1918.

[28] A. Yeganeh-Haeri, D.J. Weidner, J.B. Parise, Elasticity of alpha-cristobalite-a silicon dioxide with a negative Poisson's ratio, Science 257 (1992) 650-652.

[29] L.H. Timmins, Q. Wu, A.T. Yeh, J.E. Moore Jr., S.E. Greenwald, Structural inhomogeneity and fiber orientation in the inner arterial media, Am. J. Physiol. Heart Circ. Physiol. 298 (2010) H1537-H1545.

[30] C. Lees, J.F.V. Vincent, J.E. Hillerton, Poisson's ratio in skin, Bio-Med. Mater. Eng. 1 (1991) 19-23.

[31] K.E. Evans, Auxetic polymers: a new range of materials, Endeavor 15 (1991) 170-174.

[32] G. Fessel et al., Suitability of Thiel embalmed tendons for biomechanical investigation, Ann. Anat. 193 (2011) 237-241.

[33] A. Matson, N. Konow, S. Miller, P.P. Konow, T.J. Roberts, Tendon material properties vary and are interdependent among turkey hindlimb muscles, J. Exp. Biol. 215 (20) (2012) 3552-3558.

[34] L. Cui, H. Maas, E.J. Perreault, T.G. Sandercock, In situ estimation of tendon material properties: differences between muscles of the feline hindlimb, J. Biomech. 42 (2009) 679-685.

[35] E.M. Arruda, S. Calve, R.G. Dennis, K. Mundy, K. Baar, Regional variation of tibialis anterior tendon mechanics is lost following denervation, J. Appl. Physiol. 101 (2006) 1113-1117.

[36] L.K. Wood, E.M. Arruda, S.V. Brooks, Regional stiffening with aging in tibialis anterior tendons of mice occurs independent of changes in collagen fibril morphology, J. Appl. Phys. 111 (2011) 999-1006.

[37] N.H. Scott, The incremental bulk modulus, young's modulus and Poisson's ratio in nonlinear isotropic elasticity: physically reasonable response, Math. Mech. Solids 12 (2007) 526-542.

[38] C.W. Smith, R.J. Wootton, K.E. Evans, Interpretation of experimental data for Poisson's ratio of highly nonlinear materials, Exp. Mech. 39 (1999) 356-362.

[39] A. Rossett, L. Spadola, O. Ratib, OsiriX: an open-source software for navigating in multidimensional DICOM images, J. Digit Imaging 17 (2004) 205-216.

[40] R.E. Shadwick, Soft composites, in: J.F.V. Vincent (Ed.), Biomechanics - Materials: A Practical Approach, Oxford University Press, New York, 1992, pp. 133-164.

[41] T.A.H. Järvinen, T.L.N. Järvinen, P. Kannus, J. Laszlo, M. Järvinen, Collagen fibres of the spontaneously ruptured human tendons display decreased thickness and crimp angle, J. Orthop. Res. 22 (2014) 1303-1309.

[42] P. Sharma, N. Maffulli, Biology of tendon injury: healing, modeling and remodelling, J. Musculoskelet Neuronal Interact. 6 (2) (2006) 181-190.

[43] M. Franchi et al., Crimp morphology in relaxed and stretched rat Achilles tendon, J. Anat. 210 (1) (2007) 1-7.

[44] M. Raspanti, A. Manelli, M. Franchi, A. Ruggeri, The 3D structure of crimps in the rat Achilles tendon, Matrix Biol. 24 (2004) 503-507.

[45] J. Peltonen, N.J. Cronin, L. Stenroth, T. Finni, J. Avela, Viscoelastic properties of the Achilles tendon in vivo, SpringerPlus 2 (2013) 212.

[46] R.S. Lakes, The time-dependent Poisson's ratio of viscoelastic materials can increase or decrease, Cell Polym. 11 (1992) 466-469.

[47] J.N. Grima, R. Caruana-Gauci, Mechanical metamaterials: materials that push back, Nat. Mater. 11 (2012) 565-566. 